\title{
ESTUDO DA GASEIFICAÇÃO DE MICROALGAS EM ANÁLISE TERMOGRAVIMÉTRICA
}

\author{
C. E. FIGUEIRA ${ }^{1}$ e R. GIUDICI ${ }^{1}$ \\ ${ }^{1}$ Universidade de São Paulo, Escola Politécnica, Departamento de Engenharia Química \\ Av. Prof. Luciano Gulaberto, trav.3, No. 380, 05508-010, São Paulo, SP \\ E-mail para contato: camilaef@usp.br
}

\begin{abstract}
RESUMO - Biomassas provenientes de diferentes fontes apresentam potencial para serem convertidas em produtos químicos e combustíveis gasosos para geração de energia. As algas têm o potencial de produzir consideravelmente maiores quantidades de biomassa e de lipídios por hectare do que qualquer tipo de biomassa terrestre, com a vantagem de poderem ser cultivadas em terras marginais, portanto não competem com a produção de alimentos ou outras culturas. No presente trabalho foi estudado o processo de gaseificação da microalga Chlorella vulgaris e a cinética de reação. Os experimentos foram realizados utilizando equipamento de análise termogravimétrica acoplado a um cromatógrafo gasoso. $\mathrm{O}$ efeito dos principais parâmetros tais como massa da amostra, temperatura de reação e vazão do gás de arraste (Argônio) foram avaliados. Os gases produzidos foram analisados por cromatografia gasosa, onde os principais produtos detectados durante a gaseificação foram $\mathrm{H}_{2}, \mathrm{CO}, \mathrm{CO}_{2}$ e $\mathrm{CH}_{4}$, indicando que reações de gás de água, de oxidação e de deslocamento de água, foram predominantes.
\end{abstract}

\section{INTRODUÇÃO}

A produção de energia no século 20 foi dominada por combustíveis fósseis (carvão, petróleo e gás) que representavam ainda no início do século 21 , cerca de $80 \%$ de toda a energia produzida no mundo (WEA, 2000). No século 20, a indústria química tornou-se muito dependente do petróleo e a petroquímica assumiu um papel central, sendo em boa parte uma indústria de materiais. No início do século 21, estão crescendo pressões muito fortes para a substituição do petróleo: de um lado, a ameaça de escassez, a instabilidade de preços e a sua distribuição não uniforme, que tem causado até mesmo guerras; de outro lado, a necessidade de se desacelerar o atual processo de mudanças climáticas causadas pela atividade humana, especialmente pelas emissões de $\mathrm{CO}_{2}$, metano e óxidos de nitrogênio. Por outro lado, o atual contexto do desenvolvimento científico e tecnológico tem alguns elementos muito importantes: a emergência de novas tecnologias e a meta global de transição para uma economia sustentável, baseada em recursos renováveis. Pelo cálculo do cenário de referência, com dados do Ministério de Minas e Energia, o aumento do consumo final de eletricidade no Brasil será de mais de três vezes em pouco mais de 40 anos (445 TWh em 2007 a 1.442 TWh em 2050). No cenário da Revolução Energética, esse crescimento é reduzido. O consumo final de eletricidade atinge 1.197 TWh em 2050, ou cerca de $17 \%$ menos do que no cenário de referência. 


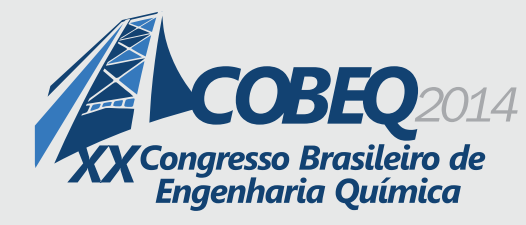

19 a 22 de outubro de 2014

Florianópolis/SC

Nos últimos anos, uma série de tentativas tem sido feitas na busca de potenciais fontes de biomassa provenientes de diferentes fontes, que podem ser convertidas em líquido, bem como combustíveis gasosos para geração de energia. Várias biomassas têm sido identificadas como fonte alternativa de combustíveis energéticos. Esta biomassa varia entre diferentes tipos de resíduos biológicos, como por exemplo, restos de comida, resíduos urbanos, resíduos agrícolas, etc; culturas energéticas, bem como as oleaginosas não comestíveis, e diversas plantas aquáticas identificadas como fontes de bio-óleo. Nos últimos anos, muito impulso foi colocado para examinar as possibilidades de utilização de algas como fonte de bio-óleo e biogás para aplicações de energia. Algas são basicamente um grupo grande e diverso de organismos simples, tipicamente autotróficos, que vão desde formas unicelulares para multicelulares. Estes têm o potencial de produzir consideravelmente maiores quantidades de biomassa e de lipídios por hectare do que qualquer tipo de biomassa terrestre. Como estas podem ser cultivadas em terras marginais, portanto não competem com alimentos ou outras culturas. As algas podem ser cultivadas fotossinteticamente utilizando a luz solar para a energia e $\mathrm{CO}_{2}$ como uma fonte de carbono. Elas podem ser cultivadas em lagoas rasas ou canais de água em terras marginais ou lagoas fechadas, precisam de menos água do que culturas terrestres (Dismukes et al., 2008).

As microalgas podem ser produzidas durante todo o ano e, por conseguinte, a quantidade de produção de óleo excede o rendimento das melhores oleaginosas, por exemplo, o rendimento de biodiesel de $58.700 \mathrm{~L} / \mathrm{ha}$ para microalgas contendo apenas $30 \%$ de óleo por peso, em comparação com 1.190 L/ha para colza ou canola (Schenk et al., 2008), 1.892 L/ha para Jatropha (Chisti, 2007), e $2.590 \mathrm{~L} / \mathrm{ha}$ para Karanj (Pongamia pinnata) (http://www.svlele.com/karanj.htm.). Há um duplo potencial para o tratamento de efluentes orgânicos a partir da indústria agroalimentar para o cultivo de algas. Além do fornecimento de meio de crescimento, os nutrientes para o cultivo, por exemplo, nitrogênio e fósforo, podem também ser obtidos a partir de águas residuais. Uma vantagem significativa ao meio ambiente é de que o cultivo de algas não necessita de aplicação de herbicidas ou pesticidas (Rodolfi et al., 2008). Além disso, elas também podem produzir valiosos coprodutos, tais como proteínas e biomassa residual depois da extração do óleo, o que pode ser utilizado como alimento ou fertilizantes, ou fermentada para produzir bioetanol ou biometano. Também as microalgas são capazes de produção foto-biológica de "bio-hidrogênio" (Ghirardi et al., 2000). Por conseguinte, torna-se imperativo que a combinação do potencial de produção de biocombustíveis, a fixação de $\mathrm{CO}_{2}$, a produção de bio-hidrogênio, e o bio-tratamento de águas residuais, como resumido acima, acentua o potencial para utilização de microalgas.

O objetivo principal deste trabalho é o de apresentar uma alternativa para a obtenção de gás de síntese unindo a utilização de uma matéria-prima pouca conhecida e utilizada (microalgas) com um processo tradicional (reação de gaseificação), mas submetido a condições inéditas de operação por acontecer em um reator solar. No entanto, optou-se primeiramente, no presente trabalho, avaliar a influência da temperatura, da massa de microalga, do fluxo de alimentação de vapor d’água e do gás de arraste (Argônio) durante a gaseificação em um equipamento de análise termogravimétrica, comparando os resultados de conversão da microalga e os rendimentos dos produtos de reação. 


\section{9 a 22 de outubro de 2014 \\ Florianópolis/SC}

\section{MATERIAIS E MÉTODOS}

\subsection{Materiais e Equipamentos Utilizados na Análise Térmica e Caracterização}

A microalga Chlorella vulgaris foi cultivada em escala de bancada no Departamento de Engenharia Química da Universidade de São Paulo (USP). A composição elementar (C, H, N) da microalga foi determinada pelo Instituto de Química da Universidade de São Paulo (USP) e é mostrada na Tabela 1. Os teores de umidade e cinza da amostra foram como 3,7\% e 4,8\%, respectivamente, obtidos por análise termogravimétrica TG. A composição mostrada corresponde aproximadamente à fórmula molecular $\mathrm{CH}_{1,814} \mathrm{O}_{0,511} \mathrm{~N}_{0,053}$.

Tabela 1: Análise Final centesimal e conteúdo mineral determinado por ICP da microalga Chlorella

\begin{tabular}{|c|c|c|c|c|}
\hline Biomassa & \multicolumn{4}{|c|}{ Análise Final (\% em peso) } \\
\hline \multirow{2}{*}{ Clorella vulgaris } & $\mathbf{C}$ & $\mathbf{H}$ & $\mathbf{N}$ & $\mathbf{O}^{*}$ \\
\cline { 2 - 5 } & 48,3 & 7,3 & 3,0 & 32,9 \\
\hline
\end{tabular}

* \% de oxigênio calculada a partir da diferença de $\mathrm{C}, \mathrm{H}$ e $\mathrm{N}$.

Os materiais utilizados durante o procedimento de análise termogravimétrica foram: 1) Amostra seca da Microalga Chlorella vulgaris (Chlorella v.) de fabricação própria (USP); 2) Água destilada e deionizada ( $\mathrm{pH}$ aproximadamente neutro); 3) Sistema borbulhador para vaporização da água (Brookfield EX-200); 4) Analisador Térmico DTG-60H da Shimadzu; 5) Balança analítica AND GH202; 6) Cadinho de alumina da Sinc do Brasil; 7) Cromatógrafo Gasoso Clarus 500 da Perkim Elmer.

\subsection{Procedimento Experimental de Análise Termogravimétrica (TGA)}

A primeira etapa na elaboração do projeto citado consiste em realizar experimentos de análise térmica, simulando as condições da reação de gaseificação, no intuito de obter os dados cinéticos necessários para elaboração de uma taxa global ou específica da reação.

Primeiramente, as amostras foram maceradas a fim de obter uma melhor homogeneização e foram separadas em cadinhos de alumina previamente identificados. O experimento da gaseificação foi feito em um aparato de TGA (DTG-60H Shimadzu). O equipamento utilizado na análise termogravimétrica é basicamente constituído por uma microbalança, um forno, termopares e um sistema de fluxo de gás. Cada amostra foi analisada pelo menos três vezes, sendo o valor médio verificado. $\mathrm{O}$ erro experimental nas medições de perda de peso e de temperatura foi de $\pm 0,5 \%$ e $\pm 2^{\circ} \mathrm{C}$, respectivamente.

Foi montado um aparato experimental utilizado para todo o processo de gaseificação incluindo a análise dos produtos de reação por cromatografia gasosa (GC Clarus 500 da Perkim Elmer). Experiências de gaseificação foram conduzidas na presença de vapor de água gerados por um sistema borbulhador. Argônio utilizado no TG no momento da etapa de gaseificação passava através do vapor 


\section{9 a 22 de outubro de 2014 \\ Florianópolis/SC}

d'água no borbulhador a uma determinada temperatura. Foram avaliados o efeito da temperatura de gaseificação, o peso inicial da amostra e a taxa de fluxo de Argônio.

A gaseificação da amostra foi efetuada em três passos:

- Secagem: a amostra foi aquecida sob uma atmosfera inerte de argônio puro de 30 a $125^{\circ} \mathrm{C}$ a uma taxa de aquecimento de $15^{\circ} \mathrm{C} / \mathrm{min}$;

- Pirólise: a amostra foi aquecida de $125^{\circ} \mathrm{C}$ para a temperatura de operação, a uma velocidade de aquecimento de $40^{\circ} \mathrm{C} / \mathrm{min}$. Argônio foi utilizado como gás de arraste;

- Gaseificação: o carvão obtido no processo de pirólise mais tarde foi gaseificado com uma mistura de gás reativo (argônio + vapor d’água) à temperatura de ensaio durante uma hora.

Após os testes preliminares, as condições estabelecidas para todos os ensaios de TG para o estudo cinético foram: 1) Temperatura do passo de gaseificação (reação): 550, 650, 750 e $850^{\circ} \mathrm{C}$; 2) Massa da amostra: 5, 10, 15 e 20 mg; 3) Atmosfera: ar sintético; 4) Vazão do gás de arraste (argônio): 50, 100, 150 e $200 \mathrm{~mL} / \mathrm{min}$ sob $25^{\circ} \mathrm{C}$ e 0,9 atm; 5) 5\% em vol. de vapor d’água no argônio. Todos os experimentos foram feitos em duplicata.

\section{RESULTADOS E DISCUSSÃO}

\subsection{Análise Termogravimétrica e Diferencial Termogravimétrica (TGA e DTG)}

Análise Térmica é um termo que abrange um grupo de técnicas nas quais uma propriedade física ou química de uma substância, ou de seus produtos de reação, é monitorada em função do tempo ou temperatura, enquanto a temperatura da amostra, sob uma atmosfera específica, é submetida a uma programação controlada. A termogravimetria (TG) avalia as mudanças de massa devido a interação com a atmosfera, vaporização e decomposição, fornecendo uma curva de variação da perda de massa da amostra em função do tempo e temperatura. De acordo com as definições, as análises térmicas são as técnicas mais comuns usadas para investigar o comportamento térmico e a cinética química (velocidade e mecanismo de reação) durante a reação de biomassa. A Figura 1 mostra as curvas de perda de massa da gaseificação da microalga Chlorella vulgaris para diferentes temperaturas de reação, massa de amostra inicial e fluxo de argônio (gás de arraste) sob uma taxa de aquecimento de $40^{\circ} \mathrm{C} / \mathrm{min}$.

De acordo com a literatura (Anastasakis e Ross, 2011; Sanchez-Silva et al., 2012; SanchezSilva et al., 2013) as curvas de termogravimetria (TG) e termogravimetria diferencial (DTG) revelaram três estágios de degradação para todas as condições de reação. Os intervalos de temperaturas destes estágios variam pouco no estudo de cada parâmetro. O primeiro estágio está associado com uma pequena perda de massa devido à desidratação (água a nível celular e externa). $\mathrm{O}$ segundo estágio representa as principais reações de formação, onde a maior parte do peso da amostra foi perdida como matéria volátil. Neste estágio observa-se o aparecimento de "ombros" que pode ser atribuídos a degradação de proteína e polissacarídeos solúveis em baixas temperaturas e em altas pode 


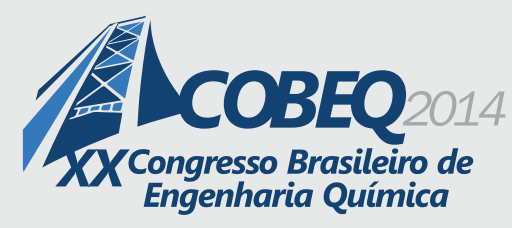

19 a 22 de outubro de 2014
Florianópolis/SC

ser atribuído a degradação da celulose na parede da célula, outros polissacarídeos insolúveis e lipídeos (Wang et al., 2007). Finalmente, o terceiro estágio representado pelas altas temperaturas está associado a formação de carvão (Marcilla at al., 2009).
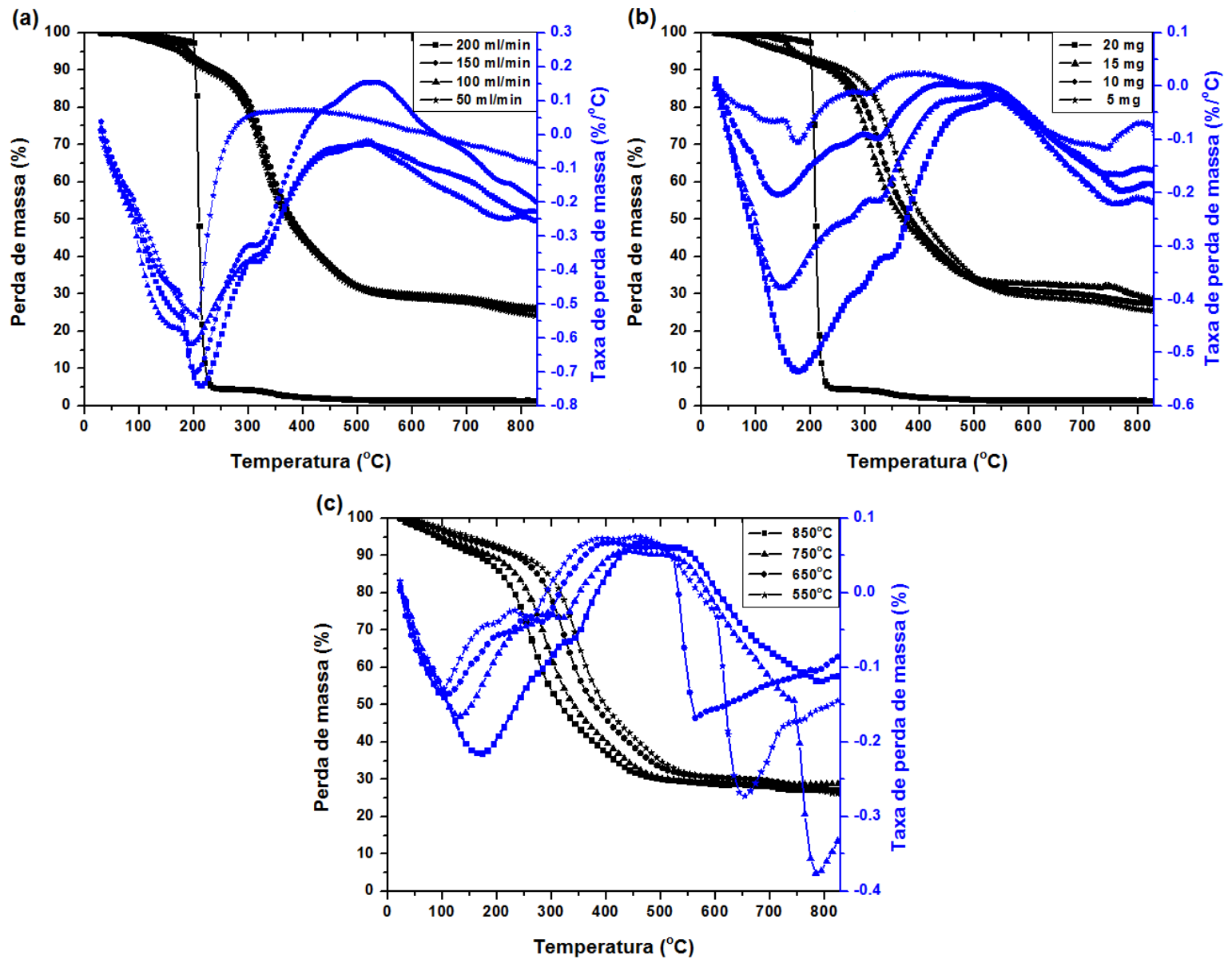

Figura 1 - Curvas termogravimétrica e diferencial termogravimétrica da gaseificação da microalga Chlorella vulgaris como função de: (a) fluxo de gás de arraste (Argônio) sob uma taxa de aquecimento de $40^{\circ} \mathrm{C} / \mathrm{min}$, (b) massa inicial de amostra e (c) temperatura de reação.

As curvas de TGA e DTG para diferentes fluxos de gás de arraste (50, 100, 150 e 200 $\mathrm{mL} / \mathrm{min})\left(25^{\circ} \mathrm{C}\right.$ e $\left.0,9 \mathrm{~atm}\right)$ usando uma massa inicial de $20 \mathrm{mg}$, temperatura de reação de $850^{\circ} \mathrm{C}$ com uma taxa de aquecimento de $40^{\circ} \mathrm{C} / \mathrm{min}$ e $5 \%$ em volume de vapor d água em argônio estão mostradas na Figura 1a. Como pode ser visto, os valores de fluxo menores que 200 não afetam os resultados da gaseificação. Dessa forma, altos fluxos de gás de arraste são requeridos a fim de evitar reações secundárias devido a longos tempos de permanência no interior do TGA.

A Figura 1b mostra as curvas de TGA e DTG em função da temperatura para o efeito da massa de amostra inicial da gaseificação da microalga. Os experimentos foram realizados usando diferentes massas iniciais (5 a 20mg), onde o fluxo de argônio (gás de arraste) foi mantido em 200 $\mathrm{mL} / \mathrm{min}\left(25^{\circ} \mathrm{C}\right.$ e $\left.0,9 \mathrm{~atm}\right)$, a temperatura de reação foi mantida em $850^{\circ} \mathrm{C}$ com uma taxa de 


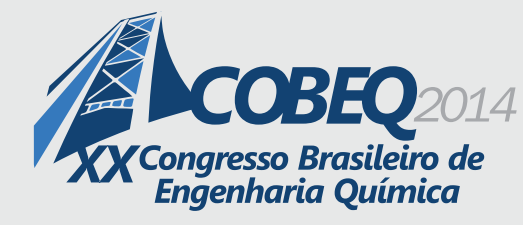

19 a 22 de outubro de 2014
Florianópolis/SC

aquecimento de $40^{\circ} \mathrm{C} / \mathrm{min}$ e $5 \%$ em volume de vapor d’água em argônio. Verificou-se que quanto maior a massa da amostra, mais pronunciados eram os eventos para cada etapa de degradação. A altura e largura dos picos DTG aumentam com o aumento do peso da amostra. No entanto, as curvas de TGA para pesos de 15, 10 e $5 \mathrm{mg}$ não se mostram muito espaçadas, quase se sobrepõem em alguns pontos, indicando limitações de transferência térmica interna e transferência de massa externa desprezíveis. Conforme descrito por Antal (1998), a altura do pico mais baixo iria corresponder com o aumento da largura de pico e um rendimento mais elevado de char. Portanto, baixas cargas de massa causam problemas de transferência de calor e massa atrasando o processo de gaseificação (Lin et al., 2009). Além disso, a forma do pico foi ligeiramente distorcida na amostra de menor massa. Com base nos resultados descritos acima, a massa da amostra inicial de $10 \mathrm{mg}$, foi selecionada para a experiência seguinte de efeito da temperatura de reação.

A Figura 1c mostra o efeito da temperatura de reação variando a mesma em 550 a $850^{\circ} \mathrm{C}$, utilizando uma massa de $10 \mathrm{mg}$, uma vazão de arraste de $200 \mathrm{ml}$ e $5 \%$ em volume de vapor d'água em Argônio. Observa-se que as temperaturas mais altas $\left(850^{\circ} \mathrm{C}\right.$ e $\left.750^{\circ} \mathrm{C}\right)$ e as temperaturas mais baixas $\left(650^{\circ} \mathrm{C}\right.$ e $\left.550^{\circ} \mathrm{C}\right)$ se sobrepõem em alguns pontos do TGA, indicando a pouca influência deste parâmetro na análise térmica de perda de massa. No entanto, observa-se que em temperaturas mais elevadas a reação se apresenta mais ativa, ou seja, a reação de gaseificação sob a maior temperatura leva um tempo menor para o início da degradação e um maior tempo para atingir o máximo de perda de massa. A diminuição da temperatura leva a uma diminuição da conversão total da microalga, que pode ser justificada pela maior formação de produtos de desidratação, quebra de ligação C-O preferencialmente em relação à quebra de ligação $\mathrm{C}-\mathrm{C}$, a baixas temperaturas, além do favorecimento à reação de deslocamento (shift). Isto poderá ser confirmado através da análise dos produtos de reação por cromatografia gasosa.

\subsection{Estudo Cinético e Quantificação dos Produtos Gasosos de Reação}

Selecionou-se a região de degradação a ser estudada nas curvas de TGA, no caso, a secagem da amostra, o começo da etapa de formação (pirólise) até o fim da análise (segunda e terceira etapas) citado anteriormente, formando o processo de gaseificação. No resultado do TGA, vários dados (variação da massa pela temperatura ou tempo) foram selecionados para satisfazer uma melhor regressão linear dada pelo método integral de Coats e Redfern (1964) (Equação 1). A sua gama de temperatura correspondente e os resultados das análises de cinética estão listados na Tabela 2.

$$
\ln \left[\frac{-\ln (1-\alpha)}{T^{2}}\right]=\ln \left[\frac{A R}{\beta E_{a}}\left(1-\frac{2 R T}{E_{a}}\right)\right]-\frac{E_{a}}{R T}
$$

onde: A é o fator pré-exponencial, $\mathrm{E}_{\mathrm{a}}$ é a energia de ativação, $\mathrm{R}$ é a constante universal dos gases, $\alpha$ é a razão de conversão da biomassa e $\beta$ representa a taxa de aquecimento da amostra. 
Tabela 2: Análise Cinética da Gaseificação da Microalga $\left(850^{\circ} \mathrm{C}, 20 \mathrm{mg}, 200 \mathrm{~mL} / \mathrm{min}\right.$, taxa de $40^{\circ} \mathrm{C} / \mathrm{min}, 5 \%$ em vol. de água em Argônio).

\begin{tabular}{|c|c|c|c|}
\hline Faixa de Temperatura $\left({ }^{\circ} \mathrm{C}\right)$ & ${\mathrm{A}\left(\mathrm{s}^{-1}\right)}^{-1}$ & $\mathrm{E}_{\mathrm{a}}(\mathrm{kJ} / \mathrm{mol})$ & $\mathrm{R}^{2}$ \\
\hline $27-200$ & $5,67.10^{-1}$ & 18,11 & 0,9745 \\
\hline $327-527$ & $1,72.10^{-1}$ & $-7,007$ & 0,9725 \\
\hline $558-850$ & $7,77.10^{-3}$ & $-10,78$ & 0,9921 \\
\hline
\end{tabular}

Todos os resultados da regressão têm bons coeficientes de determinação, $R^{2}(0,972$ 0,992). Portanto, a suposição de que a gaseificação da biomassa sofre uma reação de primeira ordem deve ser adequada. Os fatores pré-exponenciais diferem com diferentes faixas de temperatura, e variou de $5,67.10^{-1}$ a $7,77.10^{-3} \mathrm{~s}^{-1}$. Geralmente, a energia de ativação varia da mesma maneira e variou entre 18,11 e $-10,78 \mathrm{~kJ} / \mathrm{mol}$ (valores aparentes, efetivos). Embora as faixas de temperatura selecionadas sejam pequenas nas duas primeiras etapas, supõe-se que as regressões realizadas são capazes de impedir a perturbação de erros experimentais (dados de ruído) e fornecer os parâmetros cinéticos efetivos representativos nos intervalos de temperatura correspondentes. Uma vez que a composição da microalga é complicada, os parâmetros cinéticos determinados ao longo de um determinado intervalo de temperatura podem aproximar-se às de um determinado componente. No entanto, ainda se acredita que, como apontaram Vyazovkin e Wight (1998), a capacidade de um modelo matemático para produzir informações significativas não pode ser apenas caracterizada pela qualidade de ajuste.

O desempenho da reação de gaseificação da microalga Chlorella $v$. foi avaliado em condições pré-estabelecidas com os melhores valores dos parâmetros estudados (temperatura de reação, massa inicial da amostra e vazão do gás de arraste). A Tabela 3 resume o teste experimental realizado durante a gaseificação da microalga, mostrando os valores de conversão da microalga e da água, razão $\mathrm{H}_{2} / \mathrm{CO}$ e rendimento dos principais produtos obtidos durante a reação.

Tabela 3: Resumo dos resultados dos testes para a gaseificação da microalga sob $850^{\circ} \mathrm{C}, 1$ bar, 200 $\mathrm{ml} / \mathrm{min}$ e $20 \mathrm{mg}$ de microalga.

\begin{tabular}{|c|c|c|c|c|c|c|}
\hline Conversão Alga (\%) & Conversão Água (\%) & Razão $\mathrm{H}_{2} / \mathrm{CO}$ & $\mathrm{Y}_{\mathrm{H} 2}(\%)$ & $\mathrm{Y}_{\mathrm{CO}}(\%)$ & $\mathrm{Y}_{\mathrm{CO} 2}(\%)$ & $\mathrm{Y}_{\mathrm{CH} 4}(\%)$ \\
\hline 93,72 & 10,45 & 2,08 & 16,79 & 64,14 & - & 29,58 \\
\hline
\end{tabular}

Apesar de que não podemos afirmar que o estado estacionário foi obtido somente a partir da informação de que a reação de gaseificação permanece na mesma temperatura $\left(850^{\circ} \mathrm{C}\right)$ por $1 \mathrm{~h}$, essa temperatura possibilitou a formação de produtos com um átomo de carbono por molécula, tais como $\mathrm{CO}, \mathrm{CO}_{2}$ e $\mathrm{CH}_{4}$, demonstrando o favorecimento de reações com quebra de ligações $\mathrm{C}-\mathrm{C}$. 


\section{9 a 22 de outubro de 2014 \\ Florianópolis/SC}

\section{REFERÊNCIAS}

ANASTASAKIS, K.; ROSS, A. B. Hydrothermal liquefaction of the brown macroalga Laminaria saccharina: effect of reaction conditions on product distribution and composition. Bioresour. Technol., v.102 (7), p.4876-4883, 2011.

COATS, A. W.; REDFERN, J. P. Kinetic parameters from thermogravimetric data. Nature, v.201, p.68-69, 1964.

DISMUKES, G. C.; CARRIERI, D.; BENNETTE, N.; ANANYEV, G.M.; POSEWITZ, M.C. Aquatic phototrophs: efficient alternatives to land-based crops for biofuels. Curr Opin Biotechnol, v. 19, n.3, p.235-240, 2008.

GHIRARDI, M. L.; ZHANG, L.; LEE, J. W.; FLYNN, T.; SEIBERT, M.; GREENBAUM, E.; et al. Microalgae: a green source of renewable $\mathrm{H}_{2}$. Trends Biotechnol, v. 18, p. 506-511, 2000.

LIN, Y.; CHO, J.; TOMPSETT, G. A.; WESTMORELAND, P. R.; HUBER, G. W. Kinetics and mechanism of cellulose pyrolysis. J. Phys. Chem. C, v. 113, p. 20097 - 20107, 2009.

MARCILLA, A.; GÓMEZ-SIURANA, A.; GOMIS, C.; CHÁPULI, E.; CATALÁ, M. C.; VALDÉS, F. J. Characterization of microalgal species through TGTA/FTIR analysis: Application to Nannochoropsis sp. Thermochim. Acta, v.484, p.41-47, 2009.

RODOLFI, L.; ZITTELlI, G.C.; BASSI, N.; PADOVANI, G.; BIONDI, N.; BONINI, G.; et al. Microalgae for oil: strain selection, induction of lipid synthesis and outdoor mass cultivation in a lowcost photobioreactor. Biotechnol Bioeng., v.102, p.100-112, 2008.

SANCHEZ-SILVA, L.; LÓPEZ-GONZÁLEZ, D.; VILLASEÑOR, J.; SÁNCHEZ, P.; VALVERDE, J. L. Thermogravimetric mass spectrometric analysis of lignocellulosic and marine biomass pyrolysis. Bioresource Technology, v.130, p.321-331, 2012.

SANCHEZ-SILVA, L.; LÓPEZ-GONZÁLEZ, D.; GARCIA-MINGUILLAN, A. M.; VALVERDE, J. L. Pyrolysis, combustion and gasification characteristics of Nannochloropsis gaditana microalgae. Bioresource Technology, v.109, p.163-172, 2013.

SCHENK, P.; THOMAS-HALL, S.; STEPHENS, E.; MARX, U.; MUSSGNUG, J.; POSTEN, C., et al. Second generation biofuels: high-efficiency microalgae for biodiesel production. BioEnergy Res, v.1, p.20-43, 2008.

VYAZOVKIN, S.; WIGHT, C. A. Isothermal and non-isothermal kinetics of thermally stimulated reactions of solids. Int. Rev. Phys. Chem., v. 17, p. 407-433, 1998.

WANG, L.; WELlER, C. L.; JONES, D. D.; HANNA, M. A. Contemporary issues in thermal gasification of biomass and its application to electricity and fuel production. Biomass Bioenergy, v. 32, p.573-581, 2008. 